\section{Common ground in autism and epilepsy}

\section{By Lev Osherovich, Senior Writer}

A pair of studies has identified rare genomic copy number variants associated with both autism and epilepsy, suggesting there might be common mechanisms behind both diseases. ${ }^{1,2}$

The most immediate application of the findings could be to help in the early diagnosis and stratification of patients for deeper genetic studies. At the same time, the studies add support to the relatively recent hypothesis that these and other neuropsychiatric disorders like schizophrenia and mental retardation may have a common molecular basis.

Copy number variants (CNVs) are caused by DNA deletions, duplications or rearrangements scattered throughout the genome. They are usually benign but sometimes affect the activity of disease-related genes.

"Each one of us has several hundred CNVs. Some of these are common and you find differences among them in every individual, but some are rare and are more interesting from a disease perspective," said Heather Mefford, assistant professor of pediatrics and genetic medicine at the University of Washington. She was the corresponding author of the epilepsy study, which was published in PLoS Genetics.

Analyzing CNVs can identify chromosomal abnormalities that are statistically linked with disease, thus pointing to potential disease risk genes. And because of the strong biological effects caused by gross chromosomal alterations like CNVs, their analysis "has been more effective at identifying loci than genomewide association studies," noted John Spiro, senior associate director of research at the Simons Foundation Autism Research Initiative.

Geraldine Dawson, research professor of psychiatry at The University of North Carolina at Chapel Hill and CSO of the patient advocacy group Autism Speaks, agreed that the new studies confirm the importance of rare CNVs, rather than common SNPs, in neuropsychiatric indications. Dawson was a senior coauthor of the autism CNV study, which was published in Nature.

The overlapping sets of genes associated with both diseases "begs the question of whether all psychiatric diseases are along a continuum and whether there's a polygenic nature to these disorders that manifests very differently depending on what cards you're dealt," said Robert Ring, senior director and head of Pfizer Inc.'s autism research unit.
"It's really impressive that these disorders have common genetic lesions," said Ruth Ottman, professor of epidemiology and neurology at Columbia University.

Ottman noted that although many patients with autism also have epilepsy, the reverse isn't true. Thus, she said, it's surprising so many epilepsy patients appear to have CNVs in genes previously associated with autism.

"The crucial question is what is the potential link between autism and epilepsy," said Gudrun Rappold, professor in and chair of the Department of Human Molecular Genetics at Heidelberg University. "It's a puzzle that mutations in the same genes can cause very different clinical outcomes."

"There could be a number of epilepsy-specific genes or autismspecific genes and you might have to reach a certain threshold to show a clear-cut clinical phenotype," said Rappold. "It's also possible that there could be a unique combination of mutations in each individual" that lead to specific clinical conditions.

"It's clear that there are overlapping symptoms and shared biology between autism spectrum disorders, schizophrenia and affective disorders," said Ring.

If there is a common mechanism behind many neuropsychiatric diseases, the new $\mathrm{CNV}$ studies may point to pathways that could be targeted to treat any combination of these indications. But until animal models of the CNVs identified in the studies appear, it's hard to predict which targets will be most readily druggable.

-Gudrun Rappold, Heidelberg University

In addition, Spiro and Mefford noted that resequencing the candidate genes implicated by the CNVs may yet identify more subtle point mutations in the $90 \%-95 \%$ of patients in the two studies who did not carry large-scale chromosomal abnormalities. Alternatively, other genetic loci or perhaps environmental factors could prove more important in disease etiology for the majority of patients, requiring different therapeutic strategies for patients with and without CNVs.

\section{Neuron theme in autism}

The autism CNV study was done by an international consortium led by Dawson and Stephen Scherer, professor of medicine at the University of Toronto and director of The Centre for Applied Genomics at The Hospital for Sick Children.

Their team collected DNA from 1,275 children with autism spectrum disorder, and from each children's parents, and compared it with DNA from 1,981 healthy controls using a high-density microarray designed to detect CNVs. The team found 226 distinct CNVs that occurred in patients but not in controls. About $5.7 \%$ of autistic patients had at least one of the CNVs.

Many of the CNVs encompassed multiple genes, which prompted the researchers to compile a list of 149 candidate genes within the CNVs that had been linked previously to mental retardation or 
autism. These included genes for neuronal proteins involved in synaptic function.

"One of the common themes is the involvement of glutamate and the formation and function of synapses," said Dawson. She noted that the findings jibe with the theory that dysfunctional glutamate signaling underlies fragile $\mathrm{X}$ syndrome, a form of mental retardation that often is accompanied by autism. ${ }^{3}$

"This fits into our working hypothesis" about the importance of glutamate signaling in these disorders, said Randall Carpenter, president and CEO of Seaside Therapeutics Inc. "They find that relatively rare CNVs can converge on a few common signaling pathways" that have previously been linked to other neuropsychiatric indications like schizophrenia and fragile $\mathrm{X}$ syndrome.

Seaside's arbaclofen (STX209), a GABA B $_{\mathrm{B}}$ receptor agonist, is in Phase II testing for autism. The company also is developing STX109, a metabotropic glutamate receptor subtype 5 (mGluR5; GRM5) antagonist that is expected to start a Phase II trial for fragile X syndrome in early 2011. STX109 is partnered with Merck \& Co. Inc.

Dawson said the findings should help focus the attention of drug developers on signaling pathways involved in synaptic structure and function. She said Autism Speaks is organizing a conference this year to connect pharma companies with academic researchers who are developing animal models of autism related to the discoveries described in the Nature paper.

"We can now talk about how to accelerate the science from genetic discoveries to target validation," she said.

The results are unpatented.

\section{Disease nexus}

Meanwhile, Mefford's team found that many CNVs previously linked with autism, mental retardation and schizophrenia are also associated with epilepsy.

Mefford and colleagues compared genomic DNA from 517 patients with idiopathic generalized and focal epilepsy with DNA from 2,493 healthy controls. Overall, $8.9 \%$ of the epilepsy patients had one or more CNVs that were likely to account for the disease.

Twenty patients had rearrangements in several neuropsychiatric CNV hotspots, including 15 patients with CNVs near the autism-associated gene autism susceptibility candidate 2 (AUTS2).

Two other genes affected by CNVs described in Mefford's study, neurexin 1 (NRXN1) and contactin associated protein-like 2 (CNTNAP2), are also suspected autism susceptibility genes.

The most common CNV among epilepsy patients was in a region of chromosome 15 that contains the gene for nicotinic acetylcholine receptor $\alpha 7$ (CHRNA7). Mefford thinks abnormal activity of this acetylcholine receptor subunit could underlie a broad range of neuropsychiatric disorders.

Indeed, companies already are antagonizing CHRNA7 for a handful of indications. Targacept Inc. and AstraZeneca plc have TC-5619 in Phase II testing for attention deficit hyperactivity disorder (ADHD), schizophrenia and cognitive impairment. Roche's MEM 3454 (RG3487) and EnVivo Pharmaceuticals Inc.'s EVP-6124 are each in Phase II testing for
Alzheimer's disease (AD) and schizophrenia.

Mefford's next step is to make mouse knockouts that mimic the CNVs identified in her team's study. She has not patented her discoveries.

\section{Psychotic markers}

Researchers polled by SciBX agreed that the most immediate value of the two studies may be to help in the early diagnosis and stratification of patients for deeper genetic studies.

Although the specific genes altered by CNVs differed widely from patient to patient within each study, the findings nevertheless suggest that patients with similar CNVs can develop either epilepsy, autism or other neuropsychiatric disorders.

The more common of the CNVs identified in the study are "a valid basis for diagnostics and predictive screening," said Jonathan Sebat, assistant professor of psychiatry and director of the Beyster Center for Molecular Genomics of Neuropsychiatric Disease at the University of California, San Diego.

"You might hypothesize that children showing up in the clinic with deletions in some of these regions might be at risk for psychosis later in life and would be good candidates for early therapeutic intervention," Sebat said.

Dawson said that early detection and psychological therapy for autism patients can be critical in blunting the severity of behavior problems. However, she noted, because of the heterogeneous nature of the diseases caused by the CNVs, their utility as risk markers needs to be validated in prospective trials.

Osherovich, L. SciBX 3(25); doi:10.1038/scibx.2010.752

Published online June 24, 2010

REFERENCES

1. Pinto, D. et al. Nature; published online June 13, 2010; doi:10.1038/nature09146

Contact: Stephen Scherer, The Hospital for Sick Children,

Toronto, Ontario, Canada

e-mail: stephen.scherer@sickkids.ca

Contact: Geraldine Dawson, Autism Speaks, New York, N.Y. e-mail: gdawson@autismspeaks.org

2. Mefford, H.C. et al. PLoS Genet.; published online May 20, 2010; doi:10.1371/journal.pgen.1000962

Contact: Heather C. Mefford, University of Washington, Seattle, Wash. e-mail: hmefford@u.washington.edu

3. Kelleher, R.J. \& Bear, M.F. Cell 135, 401-406 (2008)

COMPANIES AND INSTITUTIONS MENTIONED

AstraZeneca plc (LSE:AZN; NYSE:AZN), London, U.K.

Autism Speaks, New York, N.Y.

Columbia University, New York, N.Y.

EnVivo Pharmaceuticals Inc., Watertown, Mass.

Heidelberg University, Heidelberg, Germany

The Hospital for Sick Children, Toronto, Ontario, Canada

Merck \& Co. Inc. (NYSE:MRK), Whitehouse Station, N.J.

Pfizer Inc. (NYSE:PFE), New York, N.Y.

Roche (SIX:ROG; OTCQX:RHHBY), Basel, Switzerland

Seaside Therapeutics Inc., Cambridge, Mass.

Simons Foundation Autism Research Initiative, New York, N.Y.

Targacept Inc. (NASDAQ:TRGT), Winston-Salem, N.C.

University of California, San Diego, Calif.

The University of North Carolina at Chapel Hill, Chapel Hill, N.C.

University of Toronto, Toronto, Ontario, Canada

University of Washington, Seattle, Wash. 